ARTIGO ORIGINAL

ORIGINAL ARTICLE

\title{
A tecnologia nos torna melhores ou apenas adia nosso dilema moral? $O$ caso dos xenotransplantes
}

\author{
Does technology make us better or only postpone our \\ moral dilemma? The case of xenotransplantation \\ Cláudio Djissey Shikida', Ari Francisco de Araujo Jr. ${ }^{2}$ \\ DOI: 10.21115/JBES.v11.n1.p18-25
}

\section{Palavras-chave: \\ transplantes, progresso tecnológico, incentivos econômicos, mercados, política pública}

\section{Keywords:}

transplantation, technological progress, economics incentives, markets, public policy

\section{RESUMO}

Objetivo: A obtenção do número adequado de órgãos cadavéricos para transplantes é um item de política pública importante. Modelos econômicos tradicionais supõem que a solução pode ser obtida por incentivos financeiros. Críticos dessa abordagem insistem que o mercado não resolve o problema, mas a intervenção estatal sim. Métodos: Este artigo apresenta o modelo do homo economicus maturus de Frey (1997), cujo principal mérito é mostrar que ambas as críticas estão incorretamente colocadas, pois indivíduos respondem não apenas a incentivos monetários de forma positiva, como demonstram os modelos tradicionais, mas também podem apresentar reações psicológicas adversas. Esse modelo é aplicado ao dilema da doação de órgãos com a inclusão do fator tecnológico, por meio da substituição de órgãos humanos por órgãos não humanos (xenotransplantes). Resultados: O principal resultado do artigo é mostrar como um avanço tecnológico pode melhorar o bem-estar sem alterar o dilema moral dos indivíduos. Conclusões: A extensão do modelo econômico tradicional permite uma análise com novas possibilidades acerca de mudanças tecnológicas e dos dilemas morais que elas podem trazer.

\begin{abstract}
Objective: Obtaining the adequate number of cadaveric organs for transplantation is an important public policy item. Traditional economic models assume that solution can be obtained by financial incentives. Critics of this approach insist that the market does not solve the problem, but state intervention does. Methods: This article presents Frey's (1997) homo economicus maturus model, whose main merit is to show that both critics are incorrectly because individuals respond not only to monetary incentives in a positive way, as traditional models demonstrate, but they can also present adverse psychological reactions. This model is applied to the dilemma of organ donation with the inclusion of the technological factor, through the replacement of human organs by non-human organs (xenotransplantation). Results: The main result of the article is to show how a technological advance can improve well-being without changing the moral dilemma of individuals. Conclusions: The extension of the traditional economic model allows an analysis with new possibilities about technological changes and the moral dilemmas that they can bring.
\end{abstract}

\footnotetext{
Recebido em: 19/11/2018. Aprovado para publicação em: 19/03/2019

1. Departamento de Economia e Programa de Pós-Graduação em Organizações e Mercados, Universidade Federal de Pelotas (UFPel), Pelotas, RS, Brasil.

2. Ibmec Minas, Belo Horizonte, MG, Brasil.

Instituição onde trabalho foi executado: Universidade Federal de Pelotas (UFPel) e lbmec Minas.

Fontes externas de financiamento: Não foram recebidos auxílios na forma de financiamento, equipamentos ou medicamentos. Congressos onde o estudo foi apresentado: $O$ estudo não foi apresentado em congressos científicos.

Conflitos de interesse: Não há potenciais conflitos de interesse.

Autor correspondente: Ari Francisco de Araujo Junior. Rua Rio Grande do Norte, 300, Funcionários, Belo Horizonte, MG, Brasil. CEP: 30130-130. Telefone: (31) 4020-5610. E-mail: arifaj@gmail.com.
} 


\section{Introdução}

"In the long run, the organ shortage may be solved with biotech miracles like transplantable animal organs genetically tailored to match individual human immune systems, or by repairing damaged organs using human stem cells. But in the short run, monetary incentives will matter." (Bailey, 2001)

"(...) selling an organ does not necessarily have to mean a commodification of the donor's body which leads to a depersonalized view of human beings and an offence against their dignity, because the question of self-degradation depends on whether the person who sells an organ evaluates his/her action as self-degradation or not." (Guttman \& Land, 1999)

O excesso de demanda de órgãos para transplantes é resolvida com incentivos de mercado ou com mais regulamentação governamental? E como desenvolvimentos biotecnológicos, como a possibilidade de transplantes interespécies (xenotransplantes) e/ou a utilização de órgãos artificiais influencia a resposta dessa pergunta? Pessoas mudam seu comportamento com o avanço da tecnologia?

Essas são as perguntas que fundamentam este artigo. Transplantes de órgãos cadavéricos humanos são um tema, na literatura brasileira, ainda distante dos economistas envolvidos em políticas públicas, embora a economia acadêmica no resto do mundo não tenha deixado de lado um tema tão caro à sociedade. Afinal, trata-se, talvez, do mais exemplar problema de alocação de recursos escassos com implicações éticas e não exclusivamente econômicas para uma sociedade.

A quantidade de órgãos cadavéricos alocada numa sociedade pode ser feita de duas formas: legal e ilegalmente. No primeiro caso, temos ainda dois mecanismos não necessariamente excludentes entre si: o mercado e o governo. Muito da literatura teórica tem se dado em direto embate de duas vertentes de pensamento (aparentemente) excludentes: pró-mercado e antimercado.

De forma sucinta, no campo da Economia, as duas estão representadas, por exemplo, por Hansman (1989), Barnett \& Kaserman (1995), Barnett II et al. (2001), Blair \& Kaserman (1991), Cohen (1995), Cohen \& Michelsen (1997), Carlstrom \& Rollow (1997), Byrne \& Thompson (2001)' e Oswald (2001). No caso de Cohen (1995), temos a proposta de um mercado futuro de órgãos, cuja discussão não é alvo deste artigo, embora bastante importante para qualquer discussão acerca

\footnotetext{
Para artigos não técnicos sobre o tema, ver, por exemplo, Becker (1997), The Economist (1997a, 1997b), Malek (2001) e Tabarrok (2002). Para uma exposição em português, ver Shikida \& Araujo Jr. (2004).
}

do uso de incentivos financeiros para se obter maior oferta de órgãos ${ }^{2}$.

Uma outra vertente da Economia que não vê, necessariamente, uma inevitável dualidade entre mercado e regulação governamental é a representada por Frey (1997), Frey \& Goette (1999) e Frey \& Jegen (2000), que se caracteriza pela formulação de um modelo que, ao contrário de negar a teoria econômica (comportamental) tradicional, amplia-a com evidências empíricas e conceitos oriundos da psicologia.

Assim, em casos como o da doação de órgãos, os incentivos financeiros poderiam desestimular os incentivos "cívicos" e, assim, levar a uma queda na oferta de órgãos doados. De forma similar para muitos autores da área médica, o uso do mecanismo de preços pode desestimular as doações ${ }^{3}$. Assim, percebe-se que o problema, embora interessante, é moralmente polêmico quando a discussão trata da escolha do mecanismo alocador de órgãos ${ }^{4}$.

\section{Objetivos}

Neste artigo, dois pontos são destacados. O primeiro diz respeito ao fato de que indivíduos podem responder de forma positiva ou negativa à adoção de um mercado ou de uma intervenção estatal mais rígida na alocação de órgãos. Isso será feito por meio de uma breve apresentação do modelo originalmente proposto por Frey (1997).

O segundo ponto é mostrar que a possibilidade de avanços tecnológicos pode resolver o problema da alocação, mas os conflitos de decisão acima aludidos permanecem intactos, vale dizer - para efeitos retóricos: avanços tecnológicos não nos tornam piores ou melhores.

\section{Métodos}

Assim, a seção seguinte apresenta o modelo citado. Em seguida, ele é ampliado com a inclusão do fator tecnológico

\footnotetext{
Dos artigos listados anteriormente, o de Byrne \& Thompson (2001) representa um contraponto específico ao caso dos incentivos financeiros defendidos pelos economistas acima citados. Contudo, o modelo trata apenas de um aspecto do problema em questão, qual seja, o de uma específica modalidade de incentivo financeiro adotada em Pittsburgh.

3 Para uma discussão dos argumentos de não economistas, veja, por exemplo, Fernandez-Montoya (1997), Houssin (1997), Nickerson et al. (1998) e Roels et al. (1997). Para uma interessante análise mista de medicina e economia, veja Obermann (1997).

4 Obviamente, neste artigo, sempre se trata da alocação legal. Uma análise do mercado ilegal, apesar de interessante e importante, não é alvo deste artigo. Várias suspeitas - baseadas apenas em entrevistas e, inclusive, com depoimentos de brasileiros - encontram-se, por exemplo, em Scheper-Hughes (1998). Entretanto, a autora parece acreditar que a escassez de órgãos para transplantes é uma invenção de "capitalistas" interessados em lucrar com essa atividade. Ainda sobre o mercado ilegal, veja, por exemplo, Rothman (1998). Para algumas "estimativas" do valor do corpo humano, veja o interessante artigo sobre as relações entre organizações não lucrativas e empresas privadas nos EUA, em Hedges \& Gaines (2000), ou a (quase)-ficcional história do leilão de órgãos no Ebay, em Prince (1999) apud Territo and Matteson (2012).
} 
(engenharia genética, clonagem ou xenotransplantes ${ }^{5}$, todos aqui chamados, resumidamente, de "xenotransplantes"). Finalmente, discutem-se as implicações do modelo, bem como suas limitações.

\section{Introdução ao Homo Economicus Maturus}

O modelo de Frey (1997) introduz a ligação da economia com a psicologia ${ }^{6}$ ao chamar a atenção para a questão do efeito crowding out sobre a ação dos indivíduos. Frey não foi o único a (re)introduzir temas correlatos de psicologia em Economia. Akerlof \& Dickens (1982) e Caplan $(2001,2003)$ são bons exemplos desse tipo de estratégia na modelagem microeconômica.

Basicamente, esse modelo considera que existem motivações intrínsecas ao indivíduo que são estimuladas ou não (respectivamente: crowding in, crowding out) conforme algum tipo de mecanismo de intervenção externa. É importante destacar que o tipo de intervenção externa não é representado apenas pelo sistema de preços (incentivos monetários), mas também pela regulação governamental'.

Obviamente, a adoção de qualquer um desses incentivos, por parte do formulador de políticas públicas, é direcionada para crowding in - ou seja, ir ao encontro das motivações intrínsecas dos indivíduos. Efeitos crowding out são indesejados, pois desestimulam os agentes, gerando, assim, reações contrárias às desejadas pelo formulador de políticas públicas. Como o modelo de Frey trata essa característica em seu modelo?

O autor parte do modelo econômico tradicional - aquele no qual um aumento do incentivo monetário/incentivo legal (coerção ou comando) gera um aumento no desempenho do indivíduo -, alterando-o de modo que a forma tomada pelo incentivo possa, ela própria, afetar a decisão do indivíduo.

Evidências sobre a relevância empírica de seu modelo são resumidas por Frey (1997) e Frey \& Jegen (2000) em problemas de mercado de trabalho (shirking, oferta de trabalho voluntária, reciprocidade, team), serviços (doação de sangue, norm adherence), bens de uso comum (dever cívico, cuidados com o meio ambiente) e problemas de Economia Política Constitucional (trust, evasão fiscal, virtudes cívicas).

5 Xenotransplantes são transplantes entre espécies. Apesar de parecerem incomuns à primeira vista, xenotransplantes são uma futura possibilidade - tal como a clonagem - para resolver o problema de escassez de órgãos para transplantes. Roth (2018) chama a atenção para o fato de que a tecnologia parece estar próxima de um ponto crítico.

6 Uma ligação, convenhamos, nada recente, tendo-se em vista as origens da Ciência Econômica.

7 Na República Popular da China, por exemplo, prisioneiros executados são, literalmente, fontes de órgãos na Ásia. Segundo Rothman (1998), médicos de vários países da Ásia: "(...) serve as travel agents, directing their patients to hospitals in Wuhan, Beijing, and Shanghai (...) Foreigners do not have to wait days or weeks for an organ to be made available; executions can be timed to meet market needs and the supply is more than adequate". [Rothman, 1998, Sec. 3]
Nessa agenda de pesquisa específica, existem, segundo Frey \& Jegen (2000), duas vertentes principais:

a) Motivações alteram preferências - A teoria de Frey (1997), na qual este artigo se baseia, permite a possibilidade de mudanças nas preferências;

b) Motivações não alteram preferências - Nesta vertente, mais fiel à hipótese tradicional de preferências fixas, tem-se, e.g., Bénabou \& Tirole (2000). O ponto central é o de que a mudança nos fatores extrínsecos afetam as motivações intrínsecas via sinalização sobre as informações que o agente processa.

O modelo de Frey pode ser resumido num sistema de agente-principal. Assim, temos:

$$
\begin{aligned}
& \text { Agente } \\
& B=B(P, E), B_{P}>0, B_{P P}<0 \\
& C=C(P, E), C_{P}>0, C_{P P}>0 \\
& L_{!}(P)=M_{P} a x B-C \\
& B=\text { benefícios (utilidade) } \\
& P=\text { quantidade/intensidade de performance } \\
& E=\text { intervenção externa } \\
& C=\text { custos }
\end{aligned}
$$

De L, obtém-se a CPO (condição de primeira ordem). Tomando-se sua diferencial total desta e rearrumando os termos, tem-se:

$$
\frac{d P^{*}}{d E}=\frac{B_{P E}-C_{P E}}{B_{P P}-C_{P P}}
$$

Note que esse é exatamente o resultado encontrado em Frey (1997). A existência do crowding out, também chamado por Frey de hidden cost of the reward (money), ocorre quando se tem $B_{P E}<0$, podendo ser reforçada quando $C_{P E}=0$, o que gera $\frac{\mathrm{dP}^{*}}{\mathrm{dE}}<0$.

Se $C_{P E}<0$ (efeito disciplinador do comando ou da recompensa monetária) e se $B_{P E}=0$, ou seja, se não existir qualquer efeito da intervenção externa sobre a performance do indivíduo, o obtido é exatamente o tradicionalmente estudado em modelos de agente-principal: $\frac{d P^{*}}{d E}>0$.

Um terceiro caso ocorre quando os dois efeitos estão presentes, i.e., $B_{P E^{\prime}} C_{P E}<0$. Nesse caso, o sinal de $\frac{d P^{*}}{d E}$ fica
indeterminado.

O principal, por sua vez, procura maximizar seu lucro líquido. Seu problema é resumido em (2), a seguir:

Principal

$\pi=\pi(\mathrm{P}), \pi_{\mathrm{p}}>0, \pi_{\mathrm{pp}}<0$

$\mathrm{K}=\mathrm{K}(\mathrm{E}), \mathrm{K}_{\mathrm{E}}>0, \mathrm{~K}_{\mathrm{EE}}>0$
$L_{2}(E)=M_{E} \pi=\pi\left(P^{*}\right)-K(E)$ 
onde:

$\pi=$ lucro

$\mathrm{K}=$ custos

De $L_{2}$, tem-se que o principal toma $P^{*}$ como um dado e escolhe o nível de $E^{*}$ ótimo. Assim, uma vez que o principal saiba o efeito de sua intervenção sobre a performance do agente, ele escolhe o nível ótimo de intervenção. Resolvendo-se a última equação de (2) usando (1a), obtém-se a CPO:

$$
\frac{d K}{d E}=\frac{d \pi}{d P} \frac{d P^{*}}{d E} \rightarrow \frac{d \pi}{d P}=\frac{d K}{d E}\left(\frac{B_{P E}-C_{P E}}{B_{P P}-C_{P P}}\right)^{-1}
$$

Dados os resultados acima, uma sugestão para a análise empírica consiste em verificar a relação $P^{*}=P(E)$, que, para o principal, pode ser interpretada como8:

"Intrinsic motivation may (...) be inferred by analyzing the behavior of the person or institution (...) administering the external intervention. Given the marginal cost of intervening, a strong reliance on external intervention by a rational actor indicates that intrinsic motivation is little or not at all affected. There might even exist a Crowding-In Effect of intervening. On the other hand, under the same condition, a low level of external intervention suggests that rational principals know that intrinsic motivation is crowded out." (Frey, 1997, p. 23)

Embora uma pesquisa possa revelar aspectos importantes da decisão individual quanto à doação de órgãos com esse modelo, os resultados podem apresentar um viés devido a não consideração de uma variável que, ao longo do tempo, tende a ser mais importante: a tecnologia de substituição de órgãos humanos por órgãos não humanos.

Assim, na seção seguinte, procura-se analisar se essa variável altera o problema de decisão, ou seja, deseja-se saber se a facilidade de uma tecnologia que altere a necessidade de órgãos humanos torna os indivíduos mais ou menos propensos à doação de órgãos.

\section{O Homo Maturus e os xenotransplantes}

"The best argument I know of against a completely free market in organs is the rent-seeking problem. (...) there would be a substantial incentive to kidnap people in order to dismember them, raising the risk of murder and the costs of defending against it. (...) One solution would be a market, but one with adequate precautions to establish chain of

8 Um exemplo de análise empírica dessa relação é Shikida et al. (2005). Neste artigo, mede-se o efeito da dissuasão moral sobre os atos de criminosos. Encontram-se evidências de que essa poderia diminuir o grau de violência dessas ações. Em outra área de pesquisa, Faria (1999) emprega uma versão dinâmica do modelo exposto para analisar problemas de Economia Política Constitucional. title to any organs sold. Another would be a legal regime in which organs could be sold by their owner, in advance or at time of death, but not resold." [Friedman, 2000, p. 242-3]

"A breakthrough came last year when researchers working separately at Nextran and at Imutran PLC in Cambridge,

England, each found a similar method to breed pigs that produce proteins that regulate human complement. In each case, human DNA was inserted into fertilized pig eggs, and the resulting swine produce both human and porcine proteins. When hearts from these pigs were transplanted into baboons and small monkeys, the organs survived far longer than did unmodified tissue. So far the longest survival time is 63 days, for a monkey treated at Imutran; the median survival time for these monkeys is 40 days." [http://wWw2.nando.net/nt/nation/, 1996]

Cientes do fato de que os xenotransplantes evoluirão no longo prazo, enquanto as necessidades sociais de órgãos transplantáveis ocorrem no curto prazo, diversos autores, conforme visto, têm proposto mecanismos de incentivos baseados no mercado para tentar aumentar o número deles.

A pergunta inevitável é: qual a influência dos xenotransplantes nas ações de consumidores? Agirão eles conforme os desejos dos elaboradores de políticas públicas ${ }^{9}$ no sentido de aumentar o número de vidas humanas "recuperadas"? Para responder a essa pergunta, o modelo deste artigo supõe que xenotransplantes sejam substitutos da necessidade de intervenção externa para a consecução de um resultado ótimo nas ações dos indivíduos.

Além disso, é bom esclarecer que a palavra "mercado", aqui, não se dissocia de uma estrutura legal de direitos de propriedade privada. Segue-se aqui argumentos como os de Cohen (1995) e Hansman (1989) [resumidos na citação de Friedman (2000) no início desta seção], sobre um mercado futuro de órgãos ${ }^{10}$, que não propugna a criação de mercados ilegais ${ }^{11}$.

Assim, novamente, considere dois indivíduos: o agente e o principal (governo). O agente pode escolher vender

9 Problemas de ação coletiva são, aqui, ignorados.

10 A melhor referência para entender a proposta de Cohen é Cohen (1995). Mas o leitor interessado em um breve resumo pode consultar Chapman (2001). Uma proposta interessante é a de Oswald (2001), que propõe um sistema de isenções tributárias para os que se comprometessem com a doação futura. Obviamente, esse sistema só seria viável se a família não pudesse interferir na retirada dos órgãos post-mortem. Além disso, qualquer tentativa do indivíduo de burlar o acordo deveria ser legalmente coibida. Caso contrário, alguns poderiam receber isenções fiscais por boa parte de sua vida e, ao fim, mudar de ideia, tornando a eficácia da proposta totalmente nula.

11 Argumentos contrários à criação de um mercado de órgãos por falta de enforcement legal sofrem da confusão entre abstração teórica e realidade. É fato que qualquer sistema econômico pode apresentar problemas de funcionamento devido a comportamentos que se desviem do que estabelece o sistema legal. Isso não invalida as conclusões de um modelo. Pelo contrário, serve como indicador de pontos que podem ser desenvolvidos em versões posteriores dele. 
seus órgãos, doá-los ou, ainda, ter seu consenso presumido, tal qual na legislação brasileira atual, a partir de $1997^{12}$. Note que a performance de um agente pode se dar, no caso do problema em análise, tanto pela venda de um órgão como pela confirmação de seu "consenso presumido", vale dizer, incentivos monetários ou de comando.

O governo - principal - garante direitos de propriedade para o funcionamento de um eventual mercado legal de órgãos cadavéricos para transplantes e gerencia os investimentos em tecnologia substituidora de órgãos humanos. Ele toma como dada a reação do agente ao mecanismo de incentivos e, então, escolhe o nível de intervenção ótima (o quanto de preço oferecer - no caso de um mercado).

Postulamos a seguinte relação entre a intervenção externa e o desenvolvimento da tecnologia de transplantes:

Tecnologia

$E=E(G) E_{G}<0$, onde

$E=$ quantidade de intervenção externa ao indivíduo

$\mathrm{G}=$ tecnologia genética

De (4) temos que avanços da tecnologia genética diminuem a necessidade de intervenção externa ao indivíduo. Argumento similar existe, por exemplo, com relação à maior intervenção do governo na área de bem-estar. Para alguns autores, o aumento da presença do Estado em atividades de bem-estar gera um efeito crowding out sobre ações de "caridade" do setor privado ${ }^{13}$.

Note que, nesse modelo, não se especifica a origem da geração da tecnologia. Pode-se supor, sem perda de generalidade, que ela seja gerada por firmas privadas.

Agente

$\mathrm{B}=\mathrm{B}(\mathrm{P}, \mathrm{E}(\mathrm{G})), \mathrm{B}_{\mathrm{P}}>0, \mathrm{~B}_{\mathrm{PP}}<0$

$C=C(P, E(G)), C_{P}>0, C_{P P}>0$

$L_{1}(P)=\operatorname{Max}_{E} B-C$

$B=$ benefícios (utilidade)

$\mathrm{P}=$ quantidade/intensidade de performance

$C=$ custos

Supõe-se que o agente não tenha qualquer controle sobre $\mathrm{G}^{14}$ e, portanto, essa variável deve ser tomada como um

12 Para breves referências sobre o caso brasileiro, veja Coelho (1997), Corrêa (2001) e o website da ABTO, citados na bibliografia.

13 Ver Andreoni \& Payne (2001) para evidências empíricas nesse sentido. A colunista Virginia Postrel, que experimentou doar um de seus órgãos, chamou a atenção para a tendência de alguns profissionais de desconfiarem de pessoas que desejam, voluntariamente, doar seus órgãos. Ver The Postrel Paradoxes Resurfaces (2006).

14 Nesse modelo o comportamento das empresas geradoras de tecnologia não é detalhado, sendo representado apenas pela equação (4). parâmetro no problema (5). De L obtém-se a CPO da qual se toma a diferencial total, obtendo-se:

$$
\frac{d P^{*}}{d E}=\frac{B_{P E}-C_{P E}}{B_{P P}-C_{P P}}
$$

Note que esse é exatamente o resultado encontrado na seção anterior. O principal, analogamente ao encontrado anteriormente, resolve o problema (6) a seguir. Para que faça sentido a análise de longo prazo, G deve, agora, ser uma variável (pode-se pensar em um argumento similar ao tradicional curto e longo prazos da teoria da firma tradicional):

$$
\begin{aligned}
& \text { Principal } \\
& \pi=\pi(P), \pi_{p}>0, \pi_{p p}<0 \\
& K=K(E(G)), K_{E}>0, K_{E E}>0 \\
& L_{2}(E)=M_{E, G} \pi=\pi\left(P^{*}\right)-K(E(G)) \\
& \text { onde: } \\
& \pi=\text { lucro } \\
& K=\text { custos }
\end{aligned}
$$

De $L_{2^{\prime}}$ vê-se que o principal toma $P^{*}$ como um dado e escolhe os níveis de $E^{*}$ e $G^{*}$ ótimos. Assim, uma vez que o principal saiba o efeito de sua intervenção sobre a performance do agente, ele escolhe o nível ótimo de intervenção. Resolvendo-se a última equação de (6), obtém-se a CPO:

$$
\frac{d K}{d E} \frac{d E}{d G}=\frac{d \pi}{d P} \frac{d P^{*}}{d E} \rightarrow \frac{d \pi}{d P}=\underbrace{\frac{d K}{d E}}_{+} \underbrace{\frac{d E}{d G}}_{?} \underbrace{\left(\frac{B_{P E}-C_{P E}}{B_{P P}-C_{P P}}\right)^{-1}}_{?}
$$

A expressão resultante em (7) nos mostra que, além da ambiguidade gerada pelo último termo do lado direito da equação visto anteriormente, surge um segundo termo relativo à substituição do incentivo pelo desenvolvimento de técnicas genéticas que diminuem a necessidade de órgãos humanos.

Observe que quanto maior o nível de G, menos intervenção se necessita. Porém, se as pessoas são motivadas apenas pelos incentivos financeiros, os ganhos de bem-estar diminuem. No caso em que as pessoas são mais "altruístas", os ganhos aumentam. Voltando ao dito de Bailey (2001) - abaixo do título da seção introdutória - sobre o curto e o longo prazo, e considerando-se que $\mathrm{G}$ é fixo no curto prazo, teríamos o resultado tradicional dado por:

$$
\frac{d \pi}{d P}=\frac{d K}{d E}\left(\frac{B_{P E}-C_{P E}}{B_{P P}-C_{P P}}\right)^{-1}
$$

Graficamente, como se ilustra o curto prazo? Na figura abaixo temos o caso em que o aumento da intervenção externa desestimula a oferta de órgãos. Quanto maior o efeito crowding out, maior o deslocamento para trás da curva de 
oferta $^{15}$. No longo prazo, contudo, qualquer uma das duas curvas de oferta (com e sem intervenção) desloca-se para baixo e para a direita, dado o efeito da tecnologia.

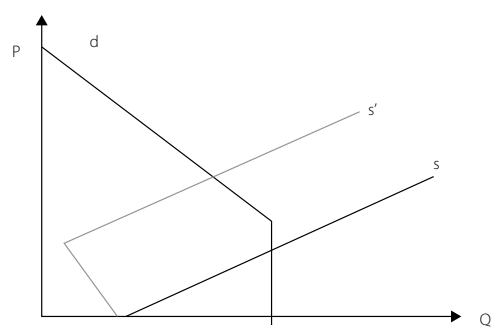

Um resumo da relação entre o curto e o longo prazo nesse modelo encontra-se no Quadro 1.

Assim, é verdade que no longo prazo a genética diminuirá a necessidade de intervenções externas. Contudo, a explicação de como isso ocorre não é tão simples como no trecho de Bailey (2001) citado no início deste artigo.

No caso (tradicional) em que vale o efeito preço - Caso A do Quadro 1 -, a engenharia genética atua fazendo com que, ao longo do tempo, exista menor necessidade de órgãos humanos. Ao final, o problema de alocação de órgãos será resolvido progressivamente com menor uso de intervenções externas e maior uso de produtos derivados da tecnologia.

No caso em que o indivíduo se sente desconfortável com a intervenção - Caso B do Quadro 1 -, mais órgãos poderão ser alocados, mas não por causa da motivação individual, e sim, apesar dela, por meio da tecnologia. Nesse caso, a performance melhora porque os custos de monitoramento caem.

Resumindo, a introdução da tecnologia mostra que o uso de intervenções (recompensas monetárias ou comando), no longo prazo, geram ganhos menores para o principal em termos de performance, mesmo que os agentes respondam positivamente ao mecanismo de preços. Caso reajam negativamente a elas, o custo de sua performance cai com o avanço tecnológico, mas a intervenção é gradativamente inócua.

Observe-se, assim, que o nível de desenvolvimento da engenharia genética (de forma geral, não apenas na forma de xenotransplantes) gera como resultado uma provável menor necessidadade de intervenção externa e, portanto, significa também um desestímulo ao mercado de órgãos humanos (embora, é bom notar, esse desestímulo seja gerado pela prosperidade do mercado para produtos biotecnológicos ${ }^{16}$.

\footnotetext{
15 A outra possibilidade é que a curva de oferta se deslocasse para trás a partir de um nível de preço positivo, ilustrando o fato de que até um certo nível de preços as pessoas não percebem o mecanismo de preços como algo "controlador". Para detalhes, veja Frey (1997), p. 108.

16 É interessante notar que a menor necessidade de intervenção externa aparece também nos argumentos pró-mercado, como em Blumstein (1989). Entretanto, o modelo apresentado neste artigo mostra que essa menor necessidade pode vir de um fator importante no longo prazo: a tecnologia. Provavelmente Blumstein e outros não se oporiam a essa afirmação, ainda que a relação entre agente e principal exposta no modelo não seja a que o autor postula.
}

O senso comum diz, em tom pessimista, que a tecnologia avança mais do que as relações humanas, em clara alusão a algum tipo de padrão moral. O modelo exposto neste artigo, de certa forma, ilustra essa tensão frequente em discussões mais normativas sobre o tema.

\section{Conclusão}

Conforme destaca Caplan (2003), assumir preferências constantes é uma hipótese bastante razoável se não existem evidências empíricas em contrário. Frey (1997) é uma tentativa de modelar situações nas quais essas mudanças ocorrem. De forma simples, o autor mostra como se pode ampliar o modelo tradicional, incluindo a possibilidade de preferências não constantes (as motivações intrínsecas dos indivíduos). Obviamente, o passo seguinte é verificar a existência empírica dessa hipótese. Talvez seja razoável pensar que, em determinados tipos de mercados, esses efeitos sejam mais fortes do que em outros. Para proponentes de políticas públicas, considerar também essa hipótese é algo bastante razoável.

Entretanto, seu modelo apresenta um trade-off que se limita à uma situação específica na qual não existe tecnologia envolvida na produção de intervenções por parte do principal. Como ficam as conclusões originais de Frey (1997) quando se introduz tecnologia em seu modelo? Neste artigo, modelou-se a tecnologia como substituta da necessidade de intervenção externa que o principal necessita exercer sobre o agente.

Empiricamente, pensa-se nos xenotransplantes como exemplo desse modelo. Sua importância, no caso específico da doação de órgãos, tem aumentado rapidamente. Por volta de 2002, o periódico escocês The Scotsman noticiava a criação de porcos clonados com o uso de DNA humano. Em 2007, divulgou-se a criação de uma ovelha "quimera", cujos órgãos seriam parcialmente humanos. A pesquisa teria como objetivo explícito auxiliar na solução de problemas de transplantes de órgãos ${ }^{17}$. Entre outros, essa inovação permitiria produzir órgãos de suínos que poderiam ser testados em seres humanos em menos de cinco anos.

Essa possibilidade, conforme visto, não altera o dilema de curto prazo do indivíduo, e os resultados do uso de incentivos para fins de aumentos na oferta de órgãos para transplantes continuam sendo ambíguos. Uma possível proposição normativa desse modelo é a de que seria mais interessante realocar parte dos recursos monetários da regulamentação da doação para o setor biotecnológico.

O próximo passo teórico consiste em ampliar e detalhar a estrutura de mercado subjacente ao problema. Já o desafio empírico é o de mensurar a resposta das pessoas aos incentivos de um hipotético mercado para órgãos.

\footnotetext{
17 Ver Calhoun (2007)
} 
Quadro 1. Curto e longo prazo

Fator comum ao curto e longo prazos

\begin{tabular}{|c|c|c|}
\hline & $\begin{array}{l}\text { Caso A: } \\
\left(\frac{B_{P E}-C_{P E}}{B_{P P}-C_{P P}}\right)>0\end{array}$ & $\begin{array}{l}\text { Caso B: } \\
\left(\frac{B_{P E}-C_{P E}}{B_{P P}-C_{P P}}\right)<0\end{array}$ \\
\hline $\begin{array}{l}\frac{\mathrm{dE}}{\mathrm{dG}}<0 \quad \text { - desenvolvimento } \\
\text { da tecnologia genética diminui o } \\
\text { nível da intervenção necessária. }\end{array}$ & $\begin{array}{l}\text { No curto prazo, a intervenção aumenta o } \\
\text { número de doações. Em conjunto com o } \\
\text { efeito tecnológico (longo prazo) no qual há } \\
\text { o desenvolvimento genético que substitui } \\
\text { órgãos humanos, verifica-se queda na } \\
\text { performance dos agentes, diminuindo o ganho } \\
\text { do principal com a performance dos agentes. }\end{array}$ & $\begin{array}{l}\text { No curto prazo, a intervenção diminui o } \\
\text { número de doações, mas, no longo prazo, } \\
\text { o desenvolvimento genético substitui os } \\
\text { órgãos humanos. Em outras palavras, há } \\
\text { menor necessidade de intervenções externas } \\
\text { para aumentar o número de transplantes. }\end{array}$ \\
\hline
\end{tabular}

\section{Agradecimentos}

Agradecemos ao professor Stefano Florissi (Programa de Pós-Graduação em Economia da Universidade Federal do Rio Grande do Sul - PPGE-UFRGS) pelo estímulo ao desenvolvimento do artigo e pelos comentários. Agradecemos também a João R. Faria (Florida Atlantic University) e a Pedro Sant'Anna (Vanderbilt University) por diversas sugestões e críticas a versões anteriores deste artigo. Erros e omissões, obviamente, são de nossa inteira responsabilidade.

\section{Referências bibliográficas}

ABTO. Legislações e Decretos. Available at: http://www.abto.org. Accessed on: 04/03/2019.

ABTO. Registro Brasileiro de Transplantes (vários números) Available at: http://www.abto.org. Accessed on: 04/03/2019.

Adams III AF, Barnett AH, Kaserman DL. Markets for organs: the question of supply. [mimeo]. s/d.

Akerlof G, Dickens W. The economic consequences of cognitive dissonance. Am Econ Rev. 1982:307-19.

Andreoni J, Payne AA. Government grants to private charities: do they crowd out or fundraising? [mimeo]. 2001.

Bacqué MC, Cambariere R. The Organ shortage: what are organ sharing organizations doing about it? Transplant Proc. 1997;29.

Bailey R. The Case for Selling Human Organs. Reason Online. 2001. Available at: http://www.reason.com/rb/rb041801.html. Accessed on: 04/03/2019.

Barnett AH, Beard TR, Kaserman DL. Scope, learning, and cross-subsidy: organ transplants in a multi-division hospital - an extension. [mimeo]. s/d.

Barnett AH, Kaserman DL. The "rush to transplant" and organ shortages. Econ Inquiry. 1995;33:506-15.

Barnett II W, Saliba M, Walker D. A free market in kidneys: efficient and equitable. Independent Rev. 2001;3(3).

Becker GS. Tio Sam pode atenuar a escassez de órgãos. Gazeta Mercantil. 1997:A-3.

Benabou R, Tirole J. Self-confidence and social interactions. NBER Working Paper Series, n. 7585. 2000. Available at: http://www.nber.org/papers/ w7585. Acessed on: 04/03/2019.

Blair RD, Kaserman DL. The economics and ethics of alternative cadaveric organ procurement policies. Yale J Regul. 1991;8(2):403-52.
Blumstein J. Government's role in organ transplantation policy. In: Blumstein J, Sloan FA, eds. Organ Transplantation Policy - issues and prospects. Durham: Duke University Press; 1989.

Byrne MM, Thompson P. A positive analysis of financial incentives for cadaveric organ donation. J Health Econ. 2001;20.

Calhoun B. It's a sheep, it's a man, it's a sheepman! Associated Content. 2007. Available at: http://www.associatedcontent.com/article/191840/its_a_ sheep_its_a_man_its_a_sheepman.html. Acessed on: 04/03/2019.

Caplan B. Rational Ignorance versus Rational Irrationality. Kyklos. 2001;54(1).

Caplan B. Stigler-Becker versus Myers-Briggs: why preference-based explanations are scientifically meaningful and empirically important. J Econ Behav Org. 2003;50:391-405.

Carlstrom CT, Rollow CD. The rationing of transplantable organs: a troubled lineup. Cato J. 1997;17(2).

Chapman S. Make organs pay off for donor's kin. Chicago Tribune. 2001.

Coelho VSP. Faltam órgãos ou falta organização? Gazeta Mercantil. 1997:A-3.

Cohen LR, Michelsen M. The efficiency/equity puzzle and the race issue in kidney allocation: a reply to Ayres et al and unos. [mimeo]. 1997.

Cohen LR. Increasing the supply of transplant organs: The virtues of an option market, Austin: R.G. Landes; 1995.

Corrêa MS. A doação educada. 2001. Available at: http://www.no.com.br. Acessed on: 04/03/2019.

Faria JR. Is there an optimal Constitution? Constitutional Political Economy. 1999;10(2).

Fernandez-Montoya A. Altruism and payment in blood donation. Transfusion Science. 1997;18(3).

Frey BS, Goette L. Does pay motivate volunteers? Institute for Empirical Research in Economics, University of Zürich, Working Paper n.7. 1999.

Frey BS, Jegen R. Motivation crowding theory: a survey of empirical evidence. J Econ Surv. 2001;15(5):589-611.

Frey BS. Not just for the money - An Economic Theory of Personal Motivation. Cheltenham: Edward Elgar Publishing; 1997.

Friedman D. Law's Order - what economics has to do with law and why it matters. Princeton: Princeton University Press; 2000.

Genetically altered pigs bred for organ transplants. Available at: http:// www2.nando.net/nt/nation/, 1996. Acessed on: 04/03/2019.

Guttman T, Land W. Ethics in living donor organ transplantation. 1999. Available at: http://www.med.uni-muenchen.de/trans/ethics.htm. Acessed on: 04/03/2019.

Hansman $\mathrm{H}$. The economics and ethics of markets for human organs. In: Blumstein J, Sloan FA, eds. Organ Transplantation Policy - issues and prospects. Durham: Duke University Press; 1989. 
Hedges SJ, Gaines W. Donor bodies milled into growing profits. Chicago Tribune. 2000.

Houssin D. Organ shortage: a public health crisis. What is the French state doing about it? Transplant Proc. 1997;29(8):3197-8.

Jefferies DE. The body as commodity: the use of markets to cure the organ deficit. Indiana Journal Global Legal Studies: 1998;5(2). Available at: http://www.law.indiana.edu/glsj/vol15/no2/13jeffer.html. Acessed on: 04/03/2019.

Malek NP. Sell your kidney, make a profit. Ludwig Von Mises Institute Daily Article. 2001. Available at: http://www.mises.org. Acessed on: 04/03/2019.

Nickerson CAE, Jasper JD, Asch DA. Comfort level, financial incentives, and consent for organ donation. Transplant Proc. 1998;30(1):155-9.

Obermann K. Some politico-economic aspects of organ shortage in transplantation medicine. Soc Sci Med. 1997;48(3).

Organselling [internet.] Available at: http://web.organselling.com. Acessed on: 04/03/2019.

Oswald A. Economic that matters: using the tax system to solve the shortage of human organs. Kyklos. 2001;54(2/3).

Prince D. Organ for Sale - Not Wurlitzer". Daily News September. 1999.

Roels L, Roelants M, Timmermans T, Hoppenbrouwers K, Pillen E, BandeKnops J. A survey on attitudes to organ donation among three generations in a country with 10 years of presumed consent legislation. Transplant Proc. 1997;29(8):3224-5.

Roth A. Xenostransplants: the (evergreen) promise of transplantable organs from pigs. Market Design. 2018. Available at: http://marketdesigner. blogspot.com/2018/11/xenotransplants-evergreen-promise-of.html. Acessed on: 04/03/2019.
Rothman DJ. The International Organ Traffic. 1998. Available at: http://www. nybooks.com/nyrev. Acessed on: 04/03/2019.

Scheper-Hughes N. Truth and Rumor on the Organ Trail. Natural History Magazine October; 1998. p. 48-57.

Shikida CD, Araujo Jr AF, Shikida PFA. A moral importa? Rev Econ Administ. 2005;4(4):415-26

Shikida CD, Araujo Jr. AF. Introdução à economia da alocação de órgãos para transplantes: direitos de propriedade, mercado de órgãos e filas de espera no Sistema Nacional de Transplantes. Estudos CEPE. 2004;(20).

Tabarrok A. The Organ Shortage: A Tragedy of the Commons? In: Tabarrok A, org. Entrepreneurial Economics - Bright Ideas from the Dismal Science. New York: Oxford University Press; 2002.

Territo L, Matteson R. The International Trafficking of Human Organs: A Multidisciplinary Perspective. Taylor \& Francis. 2012.

The Economist Buddy can you spare a lung. Economist. 1997a:19.

The Economist Organ transplants - Liver spats. Economist. 1997b:25-6.

The Postrel Paradox Resurfaces. Organomics Blog. 2006. Available at: http:// blog.organomics.org/2006/10/16/keeping-donors-safe.aspx. Acessed on: 04/03/2019.

The Scotsman. US firm clones pigs with human DNA. Available at: http:// thescotsman.co.uk. Acessed on: 04/03/2019. 